\title{
Front Matter: Volume 6422
}

, "Front Matter: Volume 6422," Proc. SPIE 6422, Sixth Symposium Optics in Industry, 642201 (30 May 2007); doi: 10.1117/12.747652

SPIE. Event: Sixth Symposium Optics in Industry, 2007, Monterrey, Mexico 


\title{
PROCEEDINGS OF SPIE
}

\section{Sixth Symposium Optics in Industry}

\author{
Julio C. Gutiérrez-Vega \\ Josué Dávila-Rodríguez \\ Carlos López-Mariscal \\ Editors
}

8-9 March 2007
Monterrey, Mexico

Organized by

Centro de Óptica, Tecnólogico de Monterrey (Mexico)

Coorganized by

Academia Mexicana de Óptica, A.C. (Mexico)

División de Óptica de la Sociedad Mexicana de Física (Mexico)

SPIE Chapter Mexico

Published by

SPIE

Volume 6422

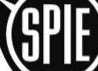


The papers included in this volume were part of the technical conference cited on the cover and title page. Papers were selected and subject to review by the editors and conference program committee. Some conference presentations may not be available for publication. The papers published in these proceedings reflect the work and thoughts of the authors and are published herein as submitted. The publisher is not responsible for the validity of the information or for any outcomes resulting from reliance thereon.

Please use the following format to cite material from this book:

Author(s), "Title of Paper," in Sixth Symposium Optics in Industry, edited by Julio C. Gutiérrez-Vega, Josué Dávila-Rodríguez, Carlos López-Mariscal, Proceedings of SPIE Vol. 6422 (SPIE, Bellingham, WA, 2007) Article CID Number.

ISSN 0277-786X

ISBN 9780819465320

Published by

SPIE

P.O. Box 10, Bellingham, Washington 98227-0010 USA

Telephone 1 360/676-3290 (Pacific Time) · Fax 1 360/647-1445

http://www.spie.org

Copyright @ 2007, The Society of Photo-Optical Instrumentation Engineers

Copying of material in this book for internal or personal use, or for the internal or personal use of specific clients, beyond the fair use provisions granted by the U.S. Copyright Law is authorized by SPIE subject to payment of copying fees. The Transactional Reporting Service base fee for this volume is $\$ 18.00$ per article (or portion thereof), which should be paid directly to the Copyright Clearance Center (CCC), 222 Rosewood Drive, Danvers, MA 01923. Payment may also be made electronically through CCC Online at http://www.copyright.com. Other copying for republication, resale, advertising or promotion, or any form of systematic or multiple reproduction of any material in this book is prohibited except with permission in writing from the publisher. The CCC fee code is 0277$786 \times / 07 / \$ 18.00$.

Printed in the United States of America.

Publication of record for individual papers is online in the SPIE Digital Library.

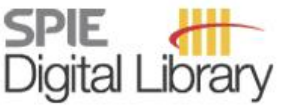

SPIEDigitalLibrary.org

Paper Numbering: Proceedings of SPIE follow an e-First publication model, with papers published firs $t$ online and then in print and on CD-ROM. Papers are published as they are submitted and meet publication criteria. A unique, consistent, permanent citation identifier (CID) number is assigned to each article at the time of the first publication. Utilization of CID numbers allows articles to be fully citable as soon they are published online, and connects the same identifier to all online, print, and electronic versions of the publication. SPIE uses a six-digit CID article numbering system in which:

- The first four digits correspond to the SPIE volume number.

- The last two digits indicate publication order within the volume using a Base 36 numbering system employing both numerals and letters. These two-number sets start with 00, 01, 02, 03, 04, 05 , $06,07,08,09,0 A, 0 B \ldots 0 Z$, followed by 10-1Z, 20-2Z, etc.

The CID number appears on each page of the manuscript. The complete citation is used on the first page, and an abbreviated version on subsequent pages. Numbers in the index correspond to the last two digits of the six-digit CID number. 


\title{
Contents
}

\author{
xi Symposium Committee \\ xiii Introduction \\ $\mathrm{xV} \quad$ Symposium Sponsors
}

\section{SESSION 1 MEDICINE AND OPHTHALMOLOGY}

642202 Irradiation of biological tissue using pulsed lasers: results and applications in medical areas [6422-01]

A. Mina-Rosales, CICESE (Mexico); G. Romo-Cárdenas, CICESE (Mexico) and CETyS Univ. (Mexico); S. Camacho-López, CICESE (Mexico); F. E. Pérez-Gutiérrez, G. Aguilar, Univ. of California, Riverside (USA); L. León-Camargo, K. P. Esparza-Moreno, Univ. Autónoma de Baja California (Mexico)

642203 Red eyes of PC users due to the effects of non-ionized electromagnetic radiation [6422-02] J. J. Soto-Bernal, Ctr. de Investigaciones en Óptica, A. C. (Mexico) and Instituto Tecnológico de Aguascalientes (Mexico); A. A. Huizar-Gonzalez, Univ. Bonaterra (Mexico); I. Rosales-Candelas, Ctr. de Investigaciones en Óptica, A. C. (Mexico) and Instituto Tecnológico de Aguascalientes (Mexico); A. R. Cardoza-Rodriguez, Instituto Tecnológico de Aguascalientes (Mexico)

642204 Genome size estimation: a new methodology [6422-03]

J. Álvarez-Borrego, CICESE (Mexico) and Univ. Autónoma de Baja California (Mexico);

C. Gallardo-Escárate, Univ. de Concepción (Chile); V. Kober, CICESE (Mexico);

O. López-Bonilla, Univ. Autónoma de Baja California (Mexico)

\section{SESSION 2 TELECOMMUNICATIONS}

642205 One application of the coherent light communication system: measurement distance with ultrasonic sensor [6422-04]

J. Paredes Jaramillo, J. Castillo Mixcóatl, G. Beltrán Pérez, S. Aguirre Muñoz,

J. A. Palma Vargas, Benemérita Univ. Autónoma de Puebla (Mexico)

642206 Novel photonics devices for optical communication systems [6422-05]

C. Calles, R. Selvas-Aguilar, A. Castillo-Guzmán, Univ. Autónoma de Nuevo León (Mexico); I. Torres-Gómez, A. Martínez-Ríos, O. Barbosa-García, Ctr. de Investigaciones en Óptica (Mexico); G. Anzueto-Sánchez, Univ. de Guanajuato (Mexico); V. Durán-Ramírez, Univ. de Guadalajara (Mexico)

642207 Reliability analysis of radio over fiber networks [6422-06]

G. Castañón, Monterrey Institute of Technology (Mexico); O. Tonguz, Carnegie Mellon Univ. (USA) 
642208 Design of a low-cost kit as a teaching tool for optical communications courses: Stage I. Basic phenomena [6422-07]

E. Alvarez Guzman, A. Nava Vega, E. Garcia Cardenas, Univ. Autonoma de Baja California (Mexico)

642209 DINAMICO [6422-08]

A. Nava Vega, J. R. Juárez Ramírez, E. Álvarez Guzmán, J. V. Ruiz López,

G. Zapata Rodríguez, Univ. Autónoma de Baja California (Mexico)

64220A Construction of a table of coordinates with optic sensors as didactic prototype [6422-09]

J. I. Hidalgo Arredondo, M. A. Moreno Barrón, M. H. Sustaita, S. E. Hernández Corpus,

P. Espinosa Padilla, M. Esparza Aranda, Polytechnic Univ. of San Luis Potosí (Mexico)

64220B Science at your school: spreading science and technology in previous educational levels [6422-10]

J.-E. Loya-Hernandez, A. Nava-Vega, J.-C. Gomez-Franco, J.-L. Gonzalez-Vazquez, Univ. Autónoma de Baja California (Mexico)

64220C Science and technology disclosure in the state of Querétaro: Science and Technology for Children program (Ciencia y Tecnología para Niños) [6422-11]

R. Contreras Flores, G. Villeda Muñoz, CICATA, Querétaro (Mexico)

\section{SESSION 4 OPTICAL MONITORING}

64220D Construction of an optical tiltmeter [6422-12]

A. Jaramillo-Núñez, M. Lucero-Alvarez, Instituto Nacional de Astrofísica, Óptica y Electrónica (Mexico)

64220E Detection of volatile organic compounds through a sensing film of $\mathrm{TiO}_{2}$ doped with organic dyes deposited on an optical fiber [6422-13]

S. Muñoz A., J. Ramos M., C. Martínez H., J. Castillo M., G. Beltrán P., R. Palomino M., Benemérita Univ. Autónoma de Puebla (Mexico)

$64220 \mathrm{~F}$ Optical techniques coupled to ohmic heating to study heat-induced changes in biological systems [6422-14]

P. A. Vázquez-Landaverde, E. Morales Sánchez, J. A. Huerta-Ruelas, Instituto Politécnico Nacional (Mexico)

$64220 \mathrm{G}$ Optical properties of a heated cornstarch mixture [6422-15]

P. A. Vazquez-Landaverde, E. Morales Sánchez, J. A. Huerta-Ruelas, Instituto Politécnico Nacional (Mexico)

$64220 \mathrm{H}$ Monitoring temperature and pressure over surfaces using sensitive paints [6422-16] J. A. Guerrero-Viramontes, D. Moreno Hernández, F. Mendoza Santoyo, Ctr. de Investigaciones en Óptica, A. C. (Mexico); J. M. Morán Loza, A. García Arreola, Univ. de Guadalajara (Mexico) 
642201 Thermal analysis of side-view mirrors [6422-17]

F. J. Gonzalez, J. D. Martinez-Ramirez, J. G. Nieto-Navarro, Univ. Autónoma de San Luis Potosí (Mexico)

64220J Application of laser interferometry to the evaluation of the dynamic characteristics of rolling bearings and comparison with piezoelectric device measurements [6422-18] D. Vela Arvizo, Univ. Autónoma del Carmen (Mexico); J. M. Rodríguez Lelis, National Ctr. for Research and Technological Development (Mexico) and Univ. Autónoma del Carmen (Mexico); M. Vargas Treviño, A. Flores Gil, M. May Alarcón, A. E. Villanueva Luna, Univ. Autónoma del Carmen (Mexico)

64220K 3D reconstruction of surface by means of modified temporal unwrapping [6422-19] J. Garzón, J. A. Galeano, C. H. López, D. A. Duque, Univ. Pontificia Bolivariana (Colombia)

64220L Shape measurement by means of fringe projection [6422-20]

D. Arroyo Almanza, B. Barrientos García, Ctr. de Investigaciones en Óptica (Mexico);

R. Martínez-Celorio, Univ. de Guanajuato (Mexico)

$64220 \mathrm{M}$ Optical fiber characterization for its implementation in speckle pattern interferometry [6422-21]

H. H. Cerecedo-Núñez, P. Padilla-Sosa, A. Sánchez-Martínez, Univ. Veracruzana (Mexico);

G. Rodríguez-Zurita, Benemérita Univ. Autónoma de Puebla (Mexico)

\section{SESSION 6 LIGHTING}

64220N Color tunable hybrid lamp: LED-incandescent and LED-fluorescent [6422-22]

I. Moreno, Univ. Autónoma de Zacatecas (Mexico) and National Central Univ. (Taiwan)

642200 Luminous flux and correlated color temperature determination for LED sources [6422-23]

A. Estrada-Hernández, L. P. González-Galván, H. Zárate-Hernández, R. Cardoso, E. Rosas, Ctr. Nacional de Metrología (Mexico)

\section{SESSION 7 LASERS AND RESONATORS}

64220P Multicavity wave resonator design using chaos studies [6422-24]

J. A. Méndez-Bermúdez, G. A. Luna-Acosta, Univ. Autónoma de Puebla (Mexico)

64220Q Optical fiber laser of multifrequency emission [6422-25]

G. Beltrán-Pérez, Benemérita Univ. Autónoma de Puebla (Mexico); L. Jacobo Aispuro,

F. Lucian Rocha, Instituto Tecnológico de Culiacán (Mexico); J. Castillo Mixcóatl,

S. Múñoz Aguirre, Benemérita Univ. Autónoma de Puebla (Mexico)

64220R Polarization controlled tunable Yb3+-doped fiber lasers [6422-26]

J. Alvarez-Chavez, A. Martinez-Rios, I. Torres-Gomez, I. Villegas-Garcia, Ctr. de Investigaciones en Óptica (Mexico); R. Rosas, Instituto Tecnológico y de Estudios Superiores de Monterrey (Mexico); H. Mercado Uribe, CINVESTAV-Monterrey (Mexico) 
$64220 S$ Observation of Mathieu-Gauss beams in axicon-based resonator [6422-27]

M. B. Alvarez-Elizondo, R. Rodríguez Y Masegosa, J. C. Gutiérrez Vega, Tecnológico de Monterrey (Mexico)

64220T Novel technique for wavelength tuning of fiber lasers [6422-28]

D. A. May-Arrioja, Instituto Nacional de Astrofísica, Óptica y Electrónica (Mexico);

R. Selvas-Aguilar, Univ. Autónoma de Nuevo Leon (Mexico); G. Anzueto-Sánchez, Univ. de Guanajuato (Mexico); A. Martínez-Rios, I. Torres-Gomez, J. Álvarez-Chávez, Ctr. de Investigaciones en Óptica (Mexico)

\section{SESSION 8 MATERIAL ANALYSIS AND TREATMENT}

$64220 \mathrm{U}$ Laser shock processing to improve residual stresses with and without paint layer on 6061-T6 aluminum alloy [6422-29]

G. Gomez-Rosas, Univ. de Guadalajara (Mexico); C. Rubio-Gonzalez, Ctr. de Ingeniería y Desarrollo Industrial (Mexico); J. L. Ocaña, C. Molpeceres, J. A. Porro, M. Morales, Univ. Politécnica de Madrid (Spain); F. J. Casillas, M. Mora-Gonzalez, Univ. de Guadalajara (Mexico); W. Chi-Moreno, Instituto Tecnológico de Morelia (Mexico)

64220V Object modelling based on laser metrology and neural networks [6422-30]

M. Rosales Ciseña, Ctr. de Ingeniería y Desarollo Industrial (Mexico);

J. Apolinar Muñoz Rodríguez, Ctr. de Investigaciones en Óptica, A.C. (Mexico);

M. Ornelas Rodríguez, Instituto Tecnológico de León (Mexico)

64220W Robust wavefront reconstruction using multiple directional derivatives and computer monitor [6422-31]

R. Legarda-Saenz, Univ. Autonoma de Yucatan (Mexico)

\section{SESSION 9 INSTRUMENTATION AND OPTICAL DEVICES}

64220X High precision prism scanning system [6422-32]

G. García-Torales, J. L. Flores, R. X. Muñoz, Univ. de Guadalajara/CUCEl (Mexico)

64220Y Detection limit of a Sagnac interferometer [6422-33]

S. Palma-Vargas, G. E. Sandoval-Romero, A. Ramírez Ibarra, Univ. Nacional Autónoma de México (Mexico)

$64220 \mathrm{O}$ Optical fiber sensor for the measurement of the $\mathrm{pH}$ level using organic dyes deposited by the sol-gel process [6422-34]

J. P. Padilla Martínez, I. Santiago Núñez, G. Beltrán Pérez, J. Castillo Mixcóatl,

S. Muñoz Aguirre, R. Palomino Merino, Benemérita Univ. Autónoma de Puebla (Mexico)

642210 Design of a zonal plate PDLC display [6422-35]

M. Pérez-Cortés, G. H. Maury-Cuna, Univ. Autónoma de Yucatán (Mexico);

M. Ortiz-Gutiérrez, Univ. Michoacana de San Nicolás de Hidalgo (Mexico);

J. Becerra-Macías, Digital Optics and Quantum Electronics (Mexico)

642211 Novel optical polarimeter using an analyzer mounted on rotator 360 stage [6422-36]

J. L. Flores, G. García-Torales, C. Rémington Juárez, M. Ernesto Cota, C. Ponce Ávila, Univ. of Guadalajara (Mexico) 
642212 Spectral birefringence characterization of monomode optical fiber devices [6422-37]

D. Tentori, Ctr. de Investigación Científica y de Educación Superior de Ensenada (Mexico);

F. Treviño-Martínez, Univ. Autónoma de Nuevo León (Mexico); C. Ayala-Díaz, Univ.

Autónoma de Baja California (Mexico); A. Gutiérrez-Beltrán, Ctr. de Investigación

Científica y de Educación Superior de Ensenada (Mexico); V. K. Carrillo-García, Ctr.

Nacional de Metrología (Mexico)

642213 Optical-integrated $\mathrm{NH}_{3}$ sensor design based on $\mathrm{WO}_{3}$ thin films: influence of gas adsorption and chromic effects [6422-38]

H. E. Lazcano Hernández, C. Sánchez Pérez, A. García Valenzuela, A. Esparza García, M. A. Camacho López, Univ. Nacional Autónoma de México (Mexico)

642214 Design and construction of a photon counting system [6422-39]

F. R. Pérez, Univ. Pontificia Bolivariana (Colombia) and Univ. de Antioquia (Colombia);

C. Del Valle, Univ. Pontificia Bolivariana (Colombia); L. Reyes, J. Tobón, C. Barrero,

A. Velásquez, Univ. de Antioquia (Colombia)

642215 Characterization of twisted liquid crystal spatial light modulators [6422-40]

U. Ruiz-Corona, V. Arrizon-Peña, Instituto Nacional de Astrofísica, Óptica y Electrónica (Mexico)

642216 Design of a high voltage source to fabricate fiber optic arc induced gratings [6422-41] R. I. Mata-Chavez, Ctr. de Investigaciones en Óptica (Mexico); J. M. Estudillo-Ayala, J. C. Hernández-Garcia, R. Rojas-Laguna, G. Anzueto-Sanchez, Univ. de Guanajuato (Mexico); A. Martínez-Ríos, Ctr. de Investigaciones en Óptica (Mexico); M. Trejo-Duran, E. A. Alvarado-Méndez, J. A. Andrade-Lucio, Univ. de Guanajuato (Mexico)

642217 Polarization-insensitive mechanically induced tunable long period holey fiber grating [6422-42]

D. E. Ceballos-Herrera, I. Torres-Gómez, E. Mejía-Beltrán, Ctr. de Investigaciones en Óptica (Mexico); R. Selvas-Aguilar, Univ. Autónoma de Nuevo León (Mexico)

\section{SESSION 10 HOLOGRAPHY}

642218 Security system with optical key access [6422-43]

C. G. Treviño-Palacios, A. Olivares-Pérez, O. J. Zapata-Nava, Instituto Nacional de Astrofísica, Óptica y Electrónica (Mexico)

642219 Implementation of complex transmittances with a phase electro-optical modulator [6422-44]

R. Carrada-Legaria, V. Arrizón, Instituto Nacional de Astrofísica, Óptica y Electrónica (Mexico)

64221 A Numerical evaluation of commercial amplitude spatial light modulator for synthesis of general complex fields [6422-45]

R. Ponce-Díaz, Instituto Tecnológico y de Estudios Superiores de Monterrey (Mexico);

V. Arrizón, Instituto Nacional de Astrofísica, Óptica y Electrónica (Mexico);

A. Serrano-Heredia, Instituto Tecnológico y de Estudios Superiores de Monterrey (Mexico) 
64221 B Optical detection of the corrosion of stainless steel 304L exposed to lithium bromide aqueous solution at $70^{\circ} \mathrm{C}$ [6422-46]

J. Castrellón-Uribe, A. Trujillo-Estrada, C. Cuevas-Arteaga, Univ. de Autónoma del Estado de Morelos (Mexico)

64221C Reflectance difference spectrometer based on the use of a CCD camera [6422-47] L. F. Lastras-Martínez, R. Castro-García, R. E. Balderas-Navarro, A. Lastras-Martínez, Univ. Autónoma de San Luis Potosí (Mexico)

64221D Absorbance and color change of LLDPE samples exposed to natural weathering in Aguascalientes City, Mexico [6422-48] R. González-Mota, Ctr. de Investigaciones en Óptica, A. C. (Mexico); J. J. Soto-Bernal, I. Rosales-Candelas, Ctr. de Investigaciones en Óptica, A. C. (Mexico) and Instituto Tecnológico de Aguascalientes (Mexico); J. T. Vega-Dúran, Instituto Politécnico Nacional (Mexico)

$64221 \mathrm{E}$ Attainment of the absorption spectra of polyacrylonitrile based on the AM1 semiempirical Hartree-Fock model [6422-49]

I. Rosales-Candelas, J. J. Soto-Bernal, Ctr. de Investigaciones en Óptica, A. C. (Mexico) and Instituto Tecnológico de Aguascalientes (Mexico); R. González Mota, Ctr. de Investigaciones en Óptica, A. C. (Mexico); J. T. Vega-Dúran, Ctr. de Investigación en Ciencia Aplicada y Tecnología Avanzada (Mexico); A. Bonilla-Petriciolet, Instituto Tecnológico de Aguascalientes (Mexico)

$64221 \mathrm{~F}$ Dispersion and refractive index measurements of diluted solutions with a double prism refractometer [6422-50]

V. Leyva-García, C. Sánchez-Pérez, A. García-Valenzuela, Univ. Nacional Autónoma de México (Mexico)

$64221 \mathrm{G} \quad$ New criterion of thin film failure of different organic coatings by shearography [6422-51] K. J. Habib, Kuwait Institute for Scientific Research (Kuwait)

$64221 \mathrm{H} \quad$ Reflectance-difference spectroscopy as an optical probe for the in situ determination of doping levels in GaAs [6422-52]

A. Lastras-Martínez, I. Lara-Velázquez, R. E. Balderas-Navarro, J. Ortega-Gallegos,

L. F. Lastras-Martínez, Univ. Autónoma de San Luis Potosí (Mexico)

\section{SESSION 12 PROCESS CONTROL}

642211 Butterfly effect on the control methods of chaos and multistability [6422-53]

J. Ventura, B. Zerega, Univ. de Guadalajara (Mexico)

$64221 \mathrm{~J} \quad$ Noise enhanced control of multistability [6422-54]

B. Zerega, Univ. de Guadalajara (Mexico); A. N. Pisarchik, Ctr. de Investigaciones en Óptica (Mexico) 
64221K Focal shift in vector Mathieu-Gauss beams [6422-55]

R. I. Hernandez-Aranda, J. C. Gutiérrez Vega, Tecnológico de Monterrey (Mexico)

64221L Nonazimuthally symmetric localized pulses in the optical domain [6422-56]

J. Davila, J. C. Gutiérrez-Vega, Tecnológico de Monterrey (Mexico)

SESSION 14 OTHER

$64221 \mathrm{M}$ Fourier transform method and temporal phase in order to measure frequency, amplitude, and surface profile of a low-frequency vibrating cantilever [6422-57]

C. Meneses-Fabian, Benemérita Univ. Autónoma de Puebla (Mexico); R. Rodriguez-Vera,

J. A. Rayas, F. Mendoza-Santoyo, Ctr. de Investigaciones en Óptica, A. C. (Mexico);

G. Rodríguez-Zurita, Benemérita Univ. Autónoma de Puebla (Mexico)

$64221 \mathrm{~N}$ Determination of the dielectric constant of coatings with a capacitance probe [6422-58]

A. Guadarrama-Santana, A. García-Valenzuela, Univ. Nacional Autónoma de México (Mexico)

642210 High Kerr optical nonlinearity observed in a liquid crystal by Z-scan technique [6422-59] R. Domínguez-Cruz, A. Padilla-Mijares, A. Méndez-Pérez, G. Romero-Galván, M. Panduro-Mendoza, Univ. Autónoma de Tamaulipas (Mexico); R. Ramos-García, Instituto Nacional de Astrofísica, Óptica y Electrónica (Mexico)

64221P Color uniformity of screens used in rear projection television [6422-60]

R. Borja, H. Márquez, CICESE (Mexico); D. Barocio, H. Millán, SONY Baja California (Mexico)

64221Q Photon electro-absorption modulation for broadband systems [6422-61]

M. Félix, Univ. Politécnica de Baja California (Mexico), Univ. Autónoma de Baja California (Mexico), and Ctr. de Investigación Científica y de Educación Superior de Ensenada (Mexico); D. Salazar, Ctr. de Investigación Científica y de Educación Superior de Ensenada (Mexico)

$64221 R$ Noise reduction of temporal phase unwrapping for 3D shape reconstruction using a quality map [6422-62]

C. A. González, A. Dávila, J. G. Garnica, Ctr. de Investigaciones en Óptica, A. C. (Mexico)

64221S Integration of InP-based photonic devices by zinc in-diffusion [6422-63]

D. A. May-Arrioja, Instituto Nacional de Astrofísica, Óptica y Electrónica (Mexico);

P. LiKamWa, The College of Optics and Photonics, Univ. of Central Florida (USA); I. Shubin,

P. YU, Univ. of California, San Diego (USA)

64221T Characterization of atomic coherence decay for storage of light experiments [6422-64] E. Figueroa, Institute for Quantum Information Science (Canada); F. Vewinger, Institute for Quantum Information Science (Canada) and Technische Univ. Kaiserslautern (Germany); J. Appel, Institute for Quantum Information Science (Canada); G. Günter, Institute for Quantum Information Science (Canada) and Univ. Konstanz (Germany); A. I. Lvovsky, Institute for Quantum Information Science (Canada) 
$64221 \mathrm{U}$ Frequency conversion and routing of quantum information in atomic media [6422-65] F. Vewinger, Institute for Quantum Information Science (Canada) and Technische Univ. Kaiserslautern (Germany); J. Appel, E. Figueroa, A. I. Lvovsky, Institute for Quantum Information Science (Canada)

$64221 \mathrm{~V}$ Static/dynamic locking range dependence on grating characteristics of index-coupled dfb lasers [6422-66]

G. Campuzano, Tecnológico de Monterrey (Mexico); P. Gallion, École Nationale Supérieure des Télécommunications (France)

$64221 \mathrm{~W}$ UV-Vis absorption spectroscopy and chemometrics to discriminate between the two basic categories and types of tequila [6422-67]

O. Barbosa Garcia, G. Ramos Ortiz, J. Pichardo Molina, J. L. Maldonado,

M. A. Meneses Nava, J. E. A. Landgrave, Ctr. de Investigaciones en Óptica, A. C. (Mexico)

Author Index 


\title{
Symposium Committee
}

\author{
Symposium Chairs
}

Julio C. Gutiérrez-Vega, Tecnológico de Monterrey (Mexico) Josué Dávila-Rodríguez, Tecnológico de Monterrey (Mexico) Carlos López-Mariscal, Tecnológico de Monterrey (Mexico)

\section{Program Committee}

Fernando Mendoza, Centro de Investigaciones en Óptica (Mexico)

Fermín Granados, Instituto Nacional de Astrofísica, Optica y Electrónica (Mexico)

Eric Rosas, Centro Nacional de Metrología (Mexico)

Diana Tentori, Centro de Investigación Científica y de Educación Superior de Ensenada (Mexico)

Rufino Díaz, Universidad Nacional Autónoma de México (Mexico) 
Downloaded From: https://www.spiedigitallibrary.org/conference-proceedings-of-spie on 26 Apr 2023

Terms of Use: https://www.spiedigitallibrary.org/terms-of-use 


\section{Introduction}

This sixth symposium on Optics in Industry has continued the effort to document ongoing work on the various facets of theory and applications of optics in industry in México. This year's conference was a two-day event.

Sixty-seven technical papers were presented, divided into fourteen topics. We believe that this division largely reflects the increasing trends and the wide applicability of optics in industrial applications. Papers from México, Canada, USA, Colombia, Peru, Spain, and Kuwait were presented.

The success of this meeting was to a great extent due to the help of the Photonics and Mathematical Optics Group at Tecnológico de Monterrey México, the SPIE Student Chapter at the same university, and the administrative staff of the Mexican Physical Society. We are grateful to the members of the organizing committee, whose shared efforts made the meeting in Monterrey fruitful and memorable. Finally, we thank also the speakers and poster presenters for their excellent presentations.

Julio C. Gutiérrez-Vega

Josué Dávila-Rodríguez Carlos López-Mariscal 
Downloaded From: https://www.spiedigitallibrary.org/conference-proceedings-of-spie on 26 Apr 2023

Terms of Use: https://www.spiedigitallibrary.org/terms-of-use 


\section{SOI Sponsorship}

Organized by:
Centro de Óptica Centro de Óptica, Tecnológico de Monterrey
Tecnológico de Monterrey

Coorganized by:

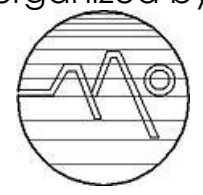

Academia Mexicana de Óptica, A.C.

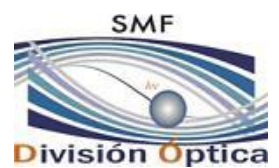

División de Óptica de la Sociedad Mexicana de Física

SPIE Chapter Mexico

With the help of:

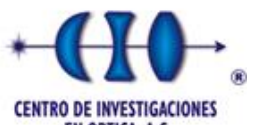
EN OPTICA, A.C.

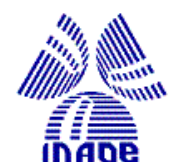

Instituto Nacional de

Astrofísica, Óptica y

Electrónica

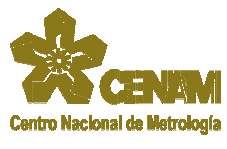

en Óptica

Centro Nacional de Matrologla

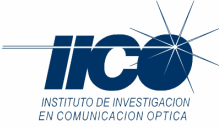

Institito de Investigación en Comunicación Óptica

Consejo Nacional de Ciencia y Tecnología
Centro Nacional de Metrología

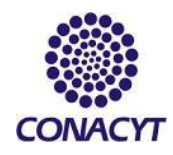

Estudios Superiores de

Ensenada

\section{clcese}

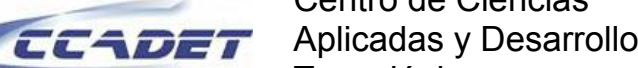

CENTRO DE CIENCIAS RPUCAPAS Y
DESARROULO TECMOLOGICO 
Sponsored by:

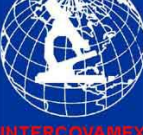

Intercovamex S.A. de C.V.

JDSU JDSU

KONICA MINOLTA

Konika Minolta Metrocal, S.A. de C.V.

[Q]METROCAL

SATELSA SATELSA

SADCA.A. Solgar S.A. de C.V.

Kodak 